\title{
Time to Turn on the Blue Lights: A Systematic Review and Meta-analysis of Photodynamic Diagnosis for Bladder Cancer
}

\author{
Rajan Veeratterapillay ${ }^{a, \dagger}$, Paul Gravestock ${ }^{a, \dagger}$, Arjun Nambiar $^{a}$, Ameet Gupta $^{a}$, \\ Omar Aboumarzouk ${ }^{a}$, Bhavan Rai ${ }^{a}$, Luke Vale ${ }^{b}$, Rakesh Heer ${ }^{a, *}$

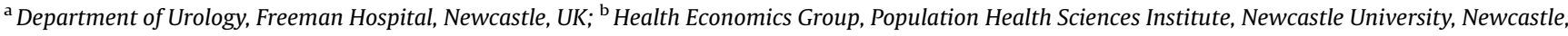

\section{Article info}

Article history:

Accepted June 28, 2021

\section{Associate Editor: Guillaume Ploussard}

Keywords:

Cystoscopy

Fluorescence

Urinary bladder neoplasms

Recurrence-free survival

Recurrence rate

\begin{abstract}
Context: White light (WL) cystoscopy and transurethral resection of bladder tumour (TURBT) comprise the current gold standard technique for detecting and grading bladder cancer. However, with WL cystoscopy, recurrence following initial TURBT is high, and identification of smaller tumours and carcinoma in situ is poor. Photodynamic diagnosis (PDD) has been developed to improve the detection of bladder.

Objective: To assess the effect of PDD-guided TURBT compared with WL on recurrence rates (RRs) in non-muscle-invasive bladder cancer (NMIBC).

Evidence acquisition: A systematic review of the literature from inception to April 2020 using Medline, EMBASE, and CENTRAL was undertaken. Randomised control trials comparing TURBT undertaken with PDD to WL that reported RRs of at least 12 mo were included in the analysis. The primary outcomes were RRs at 12 and 24 mo. The secondary outcomes were reported adverse effects. The Grading of Recommendations Assessment, Development and Evaluation (GRADE) methodology was used to assess the certainty of the evidence.

Evidence synthesis: Twelve randomised controlled trials (2288 patients) were included for the meta-analysis. PDD was found to reduce RRs at 12 mo (RR 0.73 , confidence interval [CI] 0.60-0.88) and $24 \mathrm{mo}$ (RR 0.75, CI 0.62-0.91). There was an increased risk of recurrence for patients undergoing WL at 12 mo (hazard ratio [HR] 1.14, CI 1.05-1.23) and 24 mo (HR 1.25, CI 1.15-1.35). Two studies reported recurrence data at 60 mo showing statistically significant outcomes in favour of PDD: one showed lower RRs for PDD (49\% PDD vs 68\% WL), whilst the other showed increased recurrence-free survival (68.2\% PDD vs 57.3\% WL). Adverse effects appeared to be minimal, though poorly reported. A GRADE analysis showed the evidence to be of moderate certainty overall.

Conclusions: This systematic review found that PDD reduced RRs and improved recurrence-free survival compared with WL in NMIBC over at least 2-yr follow-up.
\end{abstract}

\footnotetext{
$\uparrow$ These authors are joint first authors.

* Corresponding author. Department of Urology, Newcastle University, Newcastle, UK. Tel. 01912336161.
} 
These effects may persist up to $5 \mathrm{yr}$. Further research in a pragmatic study looking at longer-term outcomes beyond 24 mo will help guide recommendations on clinical adoption.

Patient summary: This review suggests that photodynamic diagnosis, compared with white light cystoscopy, improves recurrence-free survival in non-muscleinvasive bladder cancer over at least $2 \mathrm{yr}$ of follow-up. However, confirmatory pragmatic studies with longer-term outcomes are required for its clinical adoption.

(C) 2021 The Authors. Published by Elsevier B.V. on behalf of European Association of Urology. This is an open access article under the CC BY-NC-ND license (http://creativecommons.org/licenses/by-nc-nd/4.0/).

\section{Introduction}

Bladder cancer is the tenth commonest cancer worldwide, with an estimated 550000 cases every year [1]. The majority of bladder cancers are urothelial carcinomas that comprise muscle-invasive bladder cancer and non-muscleinvasive bladder cancer (NMIBC) [2]. At presentation, 75\% of patients have NMIBC, which histologically consist of tumours confined to the mucosa (stage pTa, carcinoma in situ [CIS]) or submucosa (pT1) but do not extend into the detrusor [3].

Currently, white light (WL) cystoscopy and transurethral resection of bladder tumour (TURBT) comprise the gold standard technique for detecting and initially staging/ grading bladder cancer. Recurrence following initial TURBT is, however, well documented and can be the result of incomplete resection of an identified tumour, missed satellite lesions, or recurrent cancer related to a diffuse premalignant field. The recurrence and progression rates can be as high as $78 \%$ and $48 \%$, respectively, in high-risk patients [4].

Furthermore, identification of smaller tumours and CIS can be poor, with up to a third of patients having further tumour noted at the first 3-mo check cystoscopy and up to half of patients developing a recurrence within $12 \mathrm{mo}$. Photodynamic diagnosis (PDD) has been developed to improve the detection of bladder tumours using fluorescence cystoscopy. Fluorescence is achieved using 5-aminolaevulinic acid (5-ALA) or its ester derivative hexaminolaevulinate (HAL), which is instilled into the bladder 1-2 h prior to cystoscopy; 5-ALA is a precursor to Protoporphyrin IX (PpIX), which is a precursor to haem in the haem-biosynthetic pathway, and an endogenous fluorophore that emits a pink-red colour when exposed to blue-violet light [5]. Following instillation of 5-ALA, PpIX preferentially accumulates within dysplastic/neoplastic tissues, which aids in their identification at blue light cystoscopy [6]. From a practical perspective, PDD is carried out in a fashion similar to traditional cystoscopy, but instead of a WL source a blue/violet light (380-440 nm) is used to illuminate the bladder wall. Following administration of the aforementioned photosensitisers, the normal bladder wall urothelium appears blue/green in colour under blue light, whereas dysplastic/malignant areas that have accumulated PpIX will emit a pink-red fluorescence ( 635 nm) [7].

PDD has been shown to have increased sensitivity in the identification of bladder tumours compared with WL, particularly of CIS lesions, in a number of studies [811]. Detection rates in one systematic review reported PDD sensitivity for biopsy-based detection of low-risk/high-risk/ CIS lesions as 96\%/99\%/86\% versus $88 \% / 67 \% / 50 \%$ in WL [12]. Another collaborative systematic review in 2013 with an expert author panel in bladder cancer recommended the use of PDD routinely, particularly for the detection of CIS lesions and to assess the completeness of TURBT [13].

Whilst it has been shown that PDD increases detection, its effect on recurrence is less clear. Initial studies showed a promising increase in recurrence-free survival (RFS); however, subsequent trials failed to replicate the earlier results, with some reporting reduced recurrence in the WL group too [14-17]. Whilst many studies have reported the impact of PDD on early recurrence rates (RRs; $<12 \mathrm{mo}$ ), medium- to long-term data ( $>12 \mathrm{mo}$ ) are less fully reported [18]. The most up-to-date systematic review by Chou et al [18] from 2016 reports RRs at $<3,3-12$, and $>12$ mo, with PDD favoured in all categories. The present study aims to provide an up-to-date systematic review and meta-analysis focussing on the effect of PDD using 5-ALA or HAL on RRs in the medium to long term. As a result, unlike previous systematic reviews that included all evidence to date, we excluded papers that reported RRs of $<12$ mo.

\section{Evidence acquisition}

\subsection{Evidence acquisition and inclusion criteria}

All randomised controlled trials (RCTs) comparing PDD (using either intravesical HAL or 5-ALA) with WL cystoscopy reporting RFS rates for at least 12 mo were considered for potential inclusion. Patient characteristics were adults $(>18$ yr) with suspected new NMIBC (any size), or those with a prior history of NMIBC and a minimum of 3 mo of recurrence-free interval, that is, not residual tumours from an incomplete resection.

\subsection{Search strategy and study selection}

This systematic review was performed in accordance with the Cochrane handbook [19] and the Preferred Reporting Items for Systematic Reviews and Meta-analyses (PRISMA) guidelines [20]. Bibliographic databases searched were Medline, EMBASE, and the Cochrane CENTRAL from inception to April 1, 2020. Search terms were used to reliably identify any relevant studies comparing PDD with 
WL in the diagnosis of bladder cancer. The search included the terms "bladder cancer", "photodynamic diagnosis", "fluorescence cystoscopy”, "5 aminolevulinic acid”, "hexyl aminolevulinate", relevant synonyms, and medical subject heading (MeSH) terms (Supplementary material). The reference lists of selected articles were reviewed to retrieve any other relevant papers that were not captured during the original search. Search results were summarised in a PRISMA flowchart.

\subsection{Outcome measures}

The primary outcomes for this review were RRs of bladder cancer at 12 and 24 mo. The secondary outcomes were adverse events as reported by trialists.

\subsection{Quality assessment of evidence}

Study quality was assessed according to the Cochrane risk of bias tool, under the domains of randomisation, allocation concealment, blinding of participants/personnel/outcome assessors, attrition bias, and selective outcome reporting
[21]. The Grading of Recommendations Assessment, Development and Evaluation (GRADE) approach was used to rate the quality of the evidence across studies for each outcome by two authors independently [22].

\subsection{Data extraction and analysis}

Studies were screened independently by two authors (R.V. and P.G.), with any disagreements arbitrated by a third author (A.N.). Data were extracted from the included studies by one author (P.G.), and reviewed and verified by another (R.V.). Variables extracted included paper and patient demographics, inclusion criteria, histology, adjuvant treatments, outcomes, and adverse effects. Comparable data from each study were combined in a meta-analysis where appropriate. An analysis was performed using a random effect model, and heterogeneity was calculated using Higgin's and Thompson's $I^{2}$ value to assess percentage of variability between studies. A subgroup analysis of 5-ALA and HAL was conducted. A further subgroup analysis based on disease characteristics was not possible due to inconsistent reporting between papers.

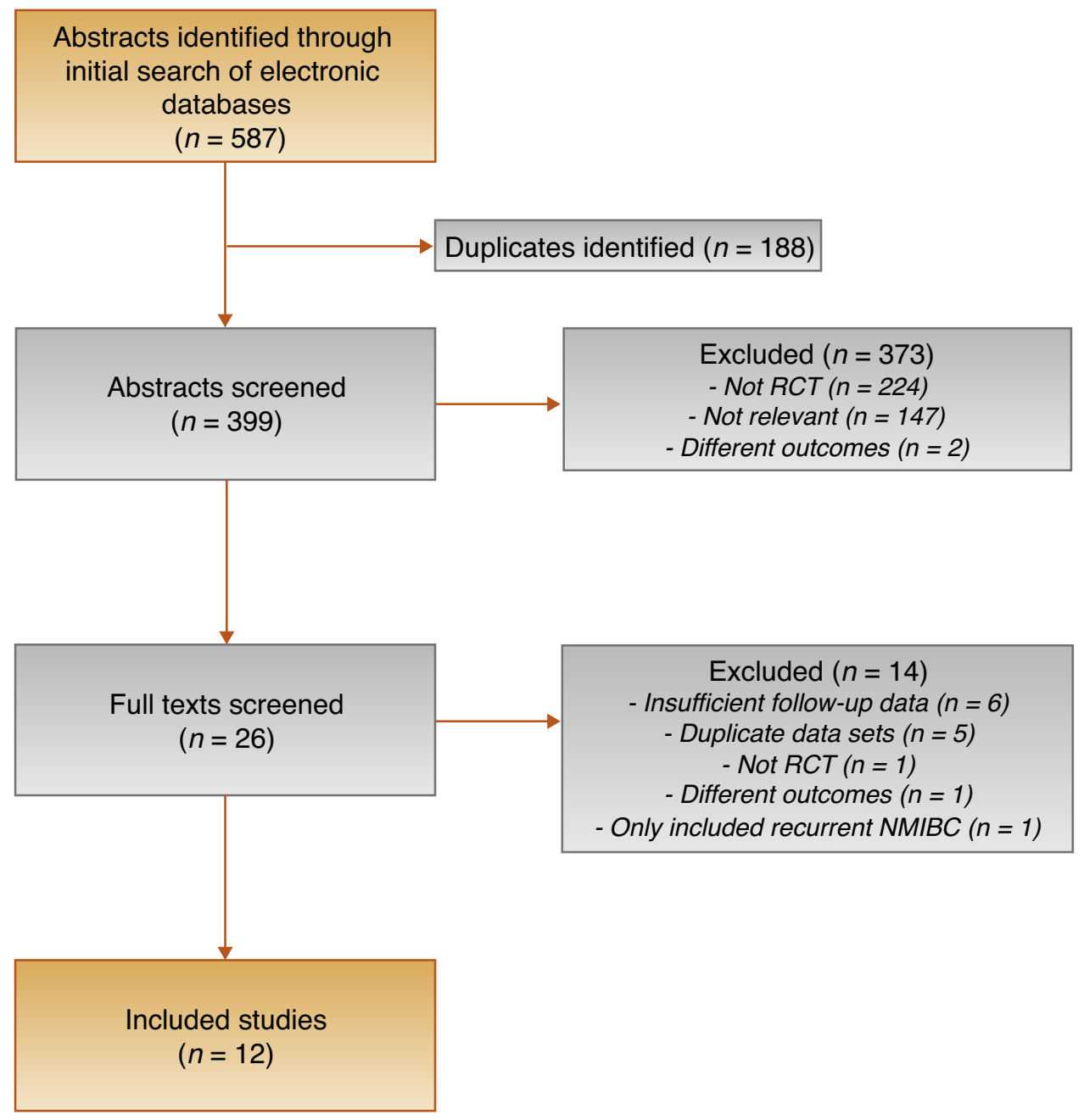

Fig. 1 - PRISMA diagram outlining the selection process. NMIBC = non-muscle-invasive bladder cancer; PRISMA = Preferred Reporting Items for Systematic Reviews and Meta-analyses; $\mathrm{RCT}=$ randomised controlled trial. 


\section{Evidence synthesis}

\subsection{Literature search}

Figure 1 shows the PRISMA diagram showing the selection of included studies. A total of 587 abstracts were identified by the search; 26 of these had their full text reviewed and 12 were deemed appropriate for inclusion.

\subsection{Study characteristics}

The study characteristics of the 12 trials (2288 patients) that met the inclusion criteria are summarised in Table 1. Of the 12 trials, half used HAL [17,23-27] and the other half used 5ALA [14,28-32], with 853 and 1430 patients, respectively. Study sample sizes ranged from 86 to 377, with follow-up between 12 and 72.3 mo. Two studies included only new presentations of bladder cancer and nine included patients with prior bladder cancer treatment with a recurrence-free interval of at least $3 \mathrm{mo}$. All studies that specified bladder cancer treatment $(n=9)$ used WL flexible cystoscopy as a surveillance modality following TURBT. Recurrence was confirmed histologically in the majority of studies $(n=6)$ $[14,23,26,28,30,32]$, one study judged recurrence by appearance \pm biopsy [17], and the remainder did not explicitly state their methodology $(n=5)$.

\subsection{Tumour characteristics}

Tumour characteristics varied considerably throughout the different patient cohorts and are summarised in Table 2. For trials that included patients with prior NMIBC, the proportion of recurrent disease at the time of enrolment varied from $18.4 \%$ [14] to $73 \%$ [26]. CIS was present in no patients in some cohorts $[17,29]$ and in up to $14.7 \%$ in others [24]. Low-risk disease was stated as provided within the studies or otherwise the criteria specified within the European Association of Urology 2020 guidelines [33] were

Table 1 - Summary of study demographics

\begin{tabular}{|c|c|c|c|c|c|c|c|c|c|c|}
\hline & \multirow[t]{2}{*}{ Author } & \multirow[t]{2}{*}{ Year } & \multirow[t]{2}{*}{$\begin{array}{l}\text { Recurrent disease } \\
\text { included? }\end{array}$} & \multicolumn{2}{|c|}{$\begin{array}{l}\text { Patient } \\
\text { cohort }(n)\end{array}$} & \multicolumn{2}{|c|}{$\begin{array}{l}\text { Mean age } \\
(\mathrm{yr})\end{array}$} & \multicolumn{2}{|c|}{ Median follow-up (mo) } & \multirow[t]{2}{*}{ Surveillance modality } \\
\hline & & & & PDD & WL & PDD & WL & PDD & WL & \\
\hline \multirow[t]{6}{*}{ HAL } & Hermann [23] & 2011 & Yes & 59 & 74 & 71 & 69 & 12 & 12 & WL \\
\hline & Geavlete [24] & 2012 & Yes & 125 & 114 & \multicolumn{2}{|c|}{66.8} & 24 & 24 & WL \\
\hline & Karaolides [25] & 2012 & Yes & 41 & 45 & 66.3 & 63.8 & $17(6-25)$ & $14(4.5-25)$ & WL \\
\hline & O'Brien [17] & 2013 & No & 97 & 88 & 68 & 68 & 12 & 12 & Not stated \\
\hline & Gkritsios [26] & 2014 & Yes & 48 & 37 & 66 & 68.2 & 40 & 40 & Not stated \\
\hline & Drăgoescu [27] & 2017 & No & 57 & 56 & 59.4 & 60.3 & $71.8^{\mathrm{a}}$ & $72.3^{a}$ & WL \\
\hline \multirow[t]{6}{*}{ 5-ALA } & Filbeck [14] & 2002 & Yes & 88 & 103 & $68^{\mathrm{b}}$ & $70^{\mathrm{b}}$ & $21(3-30)$ & $21(4-40)$ & WL \\
\hline & Babjuk [28] & 2005 & Yes & 60 & 62 & 67.9 & 69.8 & $22.4^{\mathrm{a}}$ & $20.7^{\mathrm{a}}$ & WL \\
\hline & Daniltchenko [29] & 2005 & Not stated & 51 & 51 & 70 & 67 & $42(25-61)$ & $39(23-61)$ & Not stated \\
\hline & Schumacher [30] & 2010 & Yes & 141 & 138 & 70.1 & 68.9 & 12 & 12 & $\mathrm{WL}$ \\
\hline & Stenzl [31] & 2011 & Yes & 183 & 176 & \multicolumn{2}{|c|}{66} & 12 & 12 & WL \\
\hline & Rolevich [32] & 2017 & Yes & 174 & 203 & 67 & 67 & 54 & 65 & WL \\
\hline
\end{tabular}

Table 2 - Summary of tumour characteristics

\begin{tabular}{|c|c|c|c|c|c|c|c|c|c|c|}
\hline & \multirow[t]{3}{*}{ Author } & \multirow[t]{3}{*}{ Year } & \multicolumn{8}{|c|}{ Tumour characteristics } \\
\hline & & & \multicolumn{2}{|c|}{$\begin{array}{l}\text { Proportion of } \\
\text { recurrent } \\
\text { disease }(\%)\end{array}$} & \multicolumn{2}{|c|}{ CIS (\%) } & \multicolumn{2}{|c|}{ Low-risk disease (\%) } & \multicolumn{2}{|c|}{$\begin{array}{l}\text { Multifocal } \\
\text { disease (\%) }\end{array}$} \\
\hline & & & PDD & WL & PDD & WL & PDD & WL & PDD & WL \\
\hline \multirow[t]{6}{*}{ HAL } & Hermann [23] & 2011 & \multicolumn{2}{|c|}{ No data } & \multicolumn{2}{|c|}{ No data } & 84 & 90 & 35 & 38 \\
\hline & Geavlete [24] & 2012 & \multicolumn{2}{|c|}{ No data } & 14.7 & 12.3 & $59.6(\mathrm{Ta})$ & $60.7(\mathrm{Ta})$ & 64.7 & 68 \\
\hline & Karaolides [25] & 2012 & 29.3 & 31.4 & 11.1 & 7.3 & 17.1 & 26.7 & 56.1 & 42.2 \\
\hline & O'Brien [17] & 2013 & 0 & 0 & 0 & 1.1 & 50.5 & 56.8 & 54 & 66 \\
\hline & Gkritsios [26] & 2014 & 73 & 48 & \multicolumn{2}{|c|}{ No data } & 16.7 & 16.2 & \multicolumn{2}{|c|}{ No data } \\
\hline & Drăgoescu [27] & 2017 & 0 & 0 & 5.2 & 10.5 & $28.6(\mathrm{Ta})$ & $29.8(\mathrm{Ta})$ & \multicolumn{2}{|c|}{ No data } \\
\hline \multirow[t]{6}{*}{ 5-ALA } & Filbeck [14] & 2002 & 30.7 & 18.4 & 5.7 & 4.9 & 35.2 & 48.5 & 37.5 & 23.3 \\
\hline & Babjuk [28] & 2005 & 66.6 & 54.8 & & & $63.4(\mathrm{Ta})$ & $59.7(\mathrm{Ta})$ & 75 & 63.2 \\
\hline & Daniltchenko [29] & 2005 & \multicolumn{2}{|c|}{ No data } & 0 & 1.9 & 78.4 & 78.4 & 62.8 & 39.2 \\
\hline & Schumacher [30] & 2010 & 52.5 & 50 & 0.7 & 4.3 & 54.6 & 47.8 & 46.8 & 44.2 \\
\hline & Stenzl [31] & 2011 & \multicolumn{2}{|c|}{ No data } & 1.6 & 1.7 & 33.3 & 28.4 & 43.2 & 36.4 \\
\hline & Rolevich [32] & 2017 & 34.4 & 29.5 & 1.8 & 2.5 & 15.6 & 16.3 & 55.4 & 59.1 \\
\hline
\end{tabular}


applied; in cases where insufficient data were provided, proportion of Ta disease was used as a surrogate. The proportion of low-risk disease varied from up to $90 \%$ [23] to $15.6 \%$ [32].

\subsection{Outcomes}

\subsubsection{RRs and RFS}

RRs and RFS at 12 and 24 mo for HAL and 5-ALA are presented in Table 3. Studies reporting RRs were found to have low heterogeneity $\left(\mathrm{I}^{2}=0 \%\right)$ for risk ratio calculations and those reporting RFS had moderate heterogeneity $\left(\mathrm{I}^{2}=\right.$ $70 \%$ ) for hazard ratio (HR) calculations.

For HAL at 12 mo, five trials reported RRs in the PDD group, which varied between $12.7 \%$ and $25.2 \%$ (vs $15.9 \%$ and $32.1 \%$ for WL). For 5-ALA at $12 \mathrm{mo}$, one trial reported RR in the PDD group of $30.1 \%$ (vs $37.8 \%$ for WL). At 24 mo, RRs for HAL were reported in three trials with rates for PDD between $20.0 \%$ and $29.6 \%$ (vs 27.4 and $34.9 \%$ for $\mathrm{WL}$ ). As regards 5-ALA, one trial reported RRs at 24 mo for PDD of $35.4 \%$ (vs $40 \%$ for $\mathrm{WL}$ ). A meta-analysis of $12-\mathrm{mo}$ RRs for $\mathrm{HAL}$ and 5-ALA included 833 patients across six RCTs and showed a reduction in the PDD group with an risk ratio of 0.73 (95\% confidence interval $[\mathrm{CI}] 0.60-0.88, \mathrm{I}^{2}=0 \%, p=$ 0.001 ; Fig. 2). A meta-analysis of 24-mo RRs for HAL and 5ALA included 539 patients across four RCTs and showed a reduction in the PDD group with a risk ratio of $0.75(95 \% \mathrm{CI}$ $0.62-0.91, \mathrm{I}^{2}=0 \%, p=0.003$ ) and is presented in Figure 3.

For HAL, 12- and 24-mo RFS rates with PDD were reported to range between $66.3 \%$ and $85.4 \%$ (vs $52.7 \%$ and 81.1\% for $\mathrm{WL}$ ) and between $57.9 \%$ and $75.0 \%$ (vs $46.4 \%$ and $62.2 \%$ for $\mathrm{WL}$ ), respectively. For 5-ALA, 12- and 24-mo RFS with PDD were $50.4-89.6 \%$ (vs $39.0-85.0 \%$ for $\mathrm{WL}$ ) and 40.0-89.6\% (vs 28.0-72.0\% for WL), respectively. Data were suitable for a meta-analysis of nine trials (1782 patients), and RFS at 12 mo showed an HR (in favour of PDD) of 1.14 (95\% CI 1.05-1.23, $\mathrm{I}^{2}=70 \%, p=0.002$ ), indicating that the use of PDD improved RFS. At 24 mo, a meta-analysis of five trials (925 patients) showed improved RFS following PDD (HR 1.25; 95\% CI 1.15-1.35, $p \leq 0.001$ ).

Two studies examined recurrence outcomes beyond 24 mo and reported statistically significant outcomes in favour of PDD: Drăgoescu et al [27] reported that the 24month RR following PDD was $42 \%$ versus $54 \%$ for WL, whereas at 60 mo reported RRs were $49 \%$ PDD and $68 \%$ for $\mathrm{WL}(n=113)$. A similar pattern was observed in the study by Rolevich et al [32] between RFS at long-term follow up ( $n=$ 377): PDD 68.2\% (95\% CI 59.8-76.6\%) versus WL 57.3\% (95\% CI $49.9-64.7 \%)$ at $60 \mathrm{mo}$.

\subsubsection{Adverse events}

Five studies (four using 5-ALA [14,30-32] and one using HAL [17]) involving 1391 patients reported on adverse events, whilst the remaining seven did not [23-29]. Within the studies reporting adverse events, two simply reported that no adverse events were observed [14,17], whilst one [32] reported only events requiring further surgical intervention ( $n=9$, eight endoscopic haematoma evacuations and one open bladder repair; three of nine events in the PDD group vs six in the WL group) and two studies reported all adverse events. Of all the adverse events reported by the two studies, the majority were urogenital in nature, including haematuria, dysuria, frequency/urgency, and bladder spasm $[30,31]$. One of these reported similar rates between the

Table 3 - Summary of study results

\begin{tabular}{|c|c|c|c|c|c|c|c|c|c|}
\hline & \multirow[t]{2}{*}{ Author } & \multirow[t]{2}{*}{ Year } & Adverse events & \multirow{2}{*}{$\begin{array}{l}\text { Single dose of } \\
\text { perioperative } \\
\text { intravesical } \\
\text { therapy }\end{array}$} & \multirow{2}{*}{$\begin{array}{l}\text { Period } \\
\text { (mo) }\end{array}$} & \multicolumn{2}{|c|}{ RR } & \multicolumn{2}{|c|}{ RFS (\%) } \\
\hline & & & PDD & & & PDD & WL & PDD & WL \\
\hline \multirow[t]{9}{*}{ HAL } & Hermann [23] & 2011 & Not reported & None & 12 & $18 / 59$ & $35 / 74$ & 69.5 & 52.7 \\
\hline & Geavlete [24] & 2012 & Not reported & Mitomycin C & 12 & $27 / 125$ & $37 / 114$ & 78.4 & 67.5 \\
\hline & & & & & 24 & $39 / 125$ & $52 / 114$ & 68.8 & 54.4 \\
\hline & Karaolides [25] & 2012 & Not reported & Epirubicin & 12 & Not stated & & 91 & 56.3 \\
\hline & O'Brien [17] & 2013 & None observed & Mitomycin C & 12 & $27 / 80$ & $29 / 81$ & 66.3 & 64.2 \\
\hline & Gkritsios [26] & 2014 & Not reported & Epirubicin & 12 & $7 / 48$ & $7 / 37$ & 85.4 & 81.1 \\
\hline & & & & & 24 & $12 / 48$ & $14 / 37$ & 75.0 & 62.2 \\
\hline & Drăgoescu [27] & 2017 & Not reported & Farmorubicin & 12 & $14 / 57$ & $21 / 56$ & 75.4 & 62.5 \\
\hline & & & & & 24 & $24 / 57$ & $30 / 56$ & 57.9 & 46.4 \\
\hline \multirow[t]{10}{*}{ 5-ALA } & Filbeck [14] & 2002 & None observed & None & 12 & Not stated & & 89.6 & 73.8 \\
\hline & & & & & 24 & & & 89.6 & 65.9 \\
\hline & Babjuk [28] & 2005 & Not reported & None & 12 & Not stated & & 66 & 39 \\
\hline & & & & & 24 & & & 40 & 28 \\
\hline & Daniltchenko [29] & 2005 & Not reported & None & 12 & $22 / 51$ & $31 / 51$ & 56.9 & 39.2 \\
\hline & & & & & 24 & $28 / 51$ & $34 / 51$ & 45.1 & 33.3 \\
\hline & Schumacher [30] & 2010 & $17.5 \%$ & None & 12 & Not stated & & 50.4 & 53.1 \\
\hline & Stenzl [31] & 2011 & $32.6 \% \quad 33.9 \%$ & None & 12 & Not stated & & 64 & 72.8 \\
\hline & Rolevich [32] & 2017 & Serious only: & $\sim 50 \%$ given doxorubicin & 12 & Not stated & & 89 & 85 \\
\hline & & & $1.2 \% \quad 2.2 \%$ & & 24 & & & 86 & 72 \\
\hline
\end{tabular}




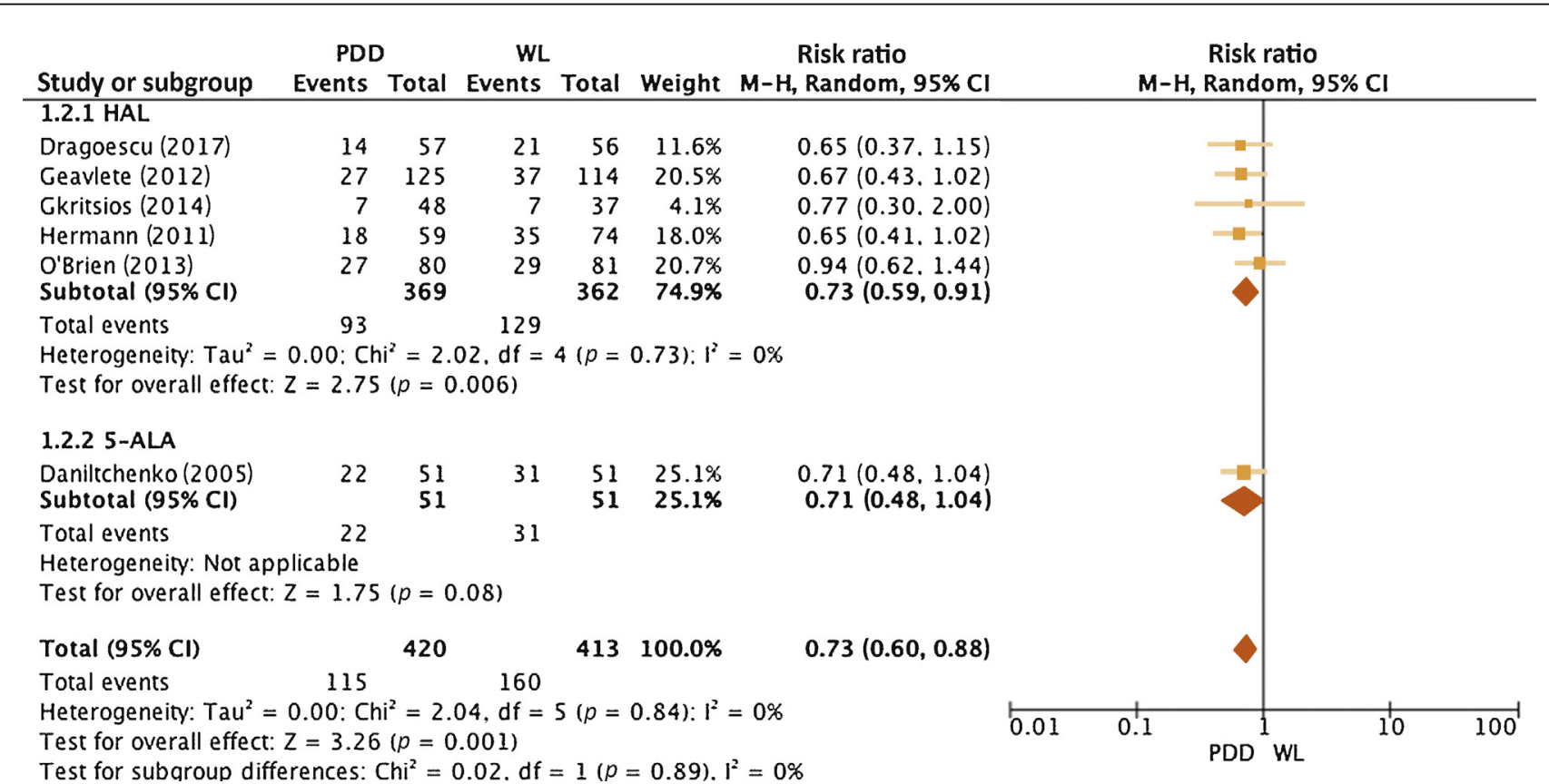

Fig. 2 - Forest plot of 12-mo recurrence rate risk ratios. 5-ALA = 5-aminolaevulinic acid; $\mathrm{CI}=$ confidence interval; $\mathbf{d f}=\mathbf{d e g r e e s}$ of freedom; $\mathrm{HAL}=$ hexaminolaevulinate; $\mathrm{M}-\mathrm{H}=$ Mantel-Haenszel; PDD = photodynamic diagnosis; $\mathrm{WL}=$ white light.

PDD Wh Risk ratio

Study or subgroup Events Total Events Total Weight M-H, Random, 95\% Cl

\begin{tabular}{lrrrrrr}
\hline 1.3.1 HAL & & & & & & \\
Dragoescu (2017) & 24 & 57 & 30 & 56 & $23.2 \%$ & $0.79(0.53,1.16)$ \\
Geavlete (2012) & 39 & 125 & 52 & 114 & $32.7 \%$ & $0.68(0.49,0.95)$ \\
Gkritsios (2014) & 12 & 48 & 14 & 37 & $8.6 \%$ & $0.66(0.35,1.25)$ \\
Subtotal (95\% Cl) & & 230 & & 207 & $\mathbf{6 4 . 5 \%}$ & $\mathbf{0 . 7 2 ( 0 . 5 7 , 0 . 9 0 )}$ \\
Total events & 75 & & 96 & & &
\end{tabular}

Heterogeneity: $\mathrm{Tau}^{2}=0.00 ; \mathrm{Chi}^{2}=0.36, \mathrm{df}=2(p=0.84) ; \mathrm{I}^{2}=0 \%$

Test for overall effect: $Z=2.80(p=0.005)$

1.3.2 5-ALA

$\begin{array}{lcccccc}\text { Daniltchenko (2005) } & 28 & 51 & 34 & 51 & 35.5 \% & 0.82(0.60,1.13) \\ \text { Subtotal (95\% Cl) } & & \mathbf{5 1} & & \mathbf{5 1} & \mathbf{3 5 . 5 \%} & \mathbf{0 . 8 2}(\mathbf{0 . 6 0}, \mathbf{1 . 1 3}) \\ \text { Total events } & 28 & & 34 & & & \end{array}$

Heterogeneity: Not applicable

Test for overall effect: $Z=1.21(p=0.23)$

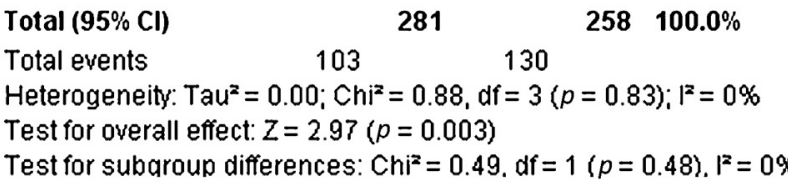

$0.75(0.62,0.91)$

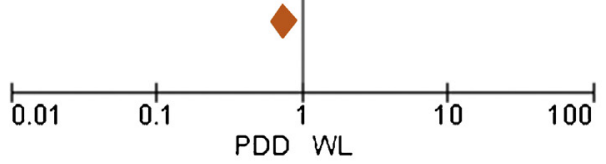

Fig. 3 - Forest plot of 24 -mo recurrence rate risk ratio. 5 -ALA = 5-aminolaevulinic acid; $\mathrm{CI}=$ confidence interval; $\mathbf{d f}=\mathbf{d e g r e e s}$ of freedom; $\mathrm{HAL}=$ hexaminolaevulinate; $\mathrm{M}-\mathrm{H}=$ Mantel-Haenszel; $\mathrm{PDD}=$ photodynamic diagnosis; $\mathrm{WL}=$ white light.

PDD and WL groups, whilst the other study showed adverse events appeared to occur more often in the PDD group $(28 \%$ vs $17.5 \%$ ) [30], but did not comment on why the authors thought this was the case. This is at odds with the other papers reporting adverse effects, which showed no difference, and with previous safety analyses of HAL, which also show no or very minimal additional risk of PDD versus WL cystoscopy [34].
3.4.3. Effect of intravesical chemotherapy and BCG

In five of the included studies, all patients received a single dose of intravesical chemotherapy (IC) following TURBT [17,24-27]. IC agents used included mitomycin C $(n=2)$, epirubicin $(n=2)$, and farmorubicin $(n=1)$. One further study by Rolevich et al [32] had four arms with combinations of WL cystoscopy or PDD with or without doxorubicin, and approximately half of patients (193/377) received a 


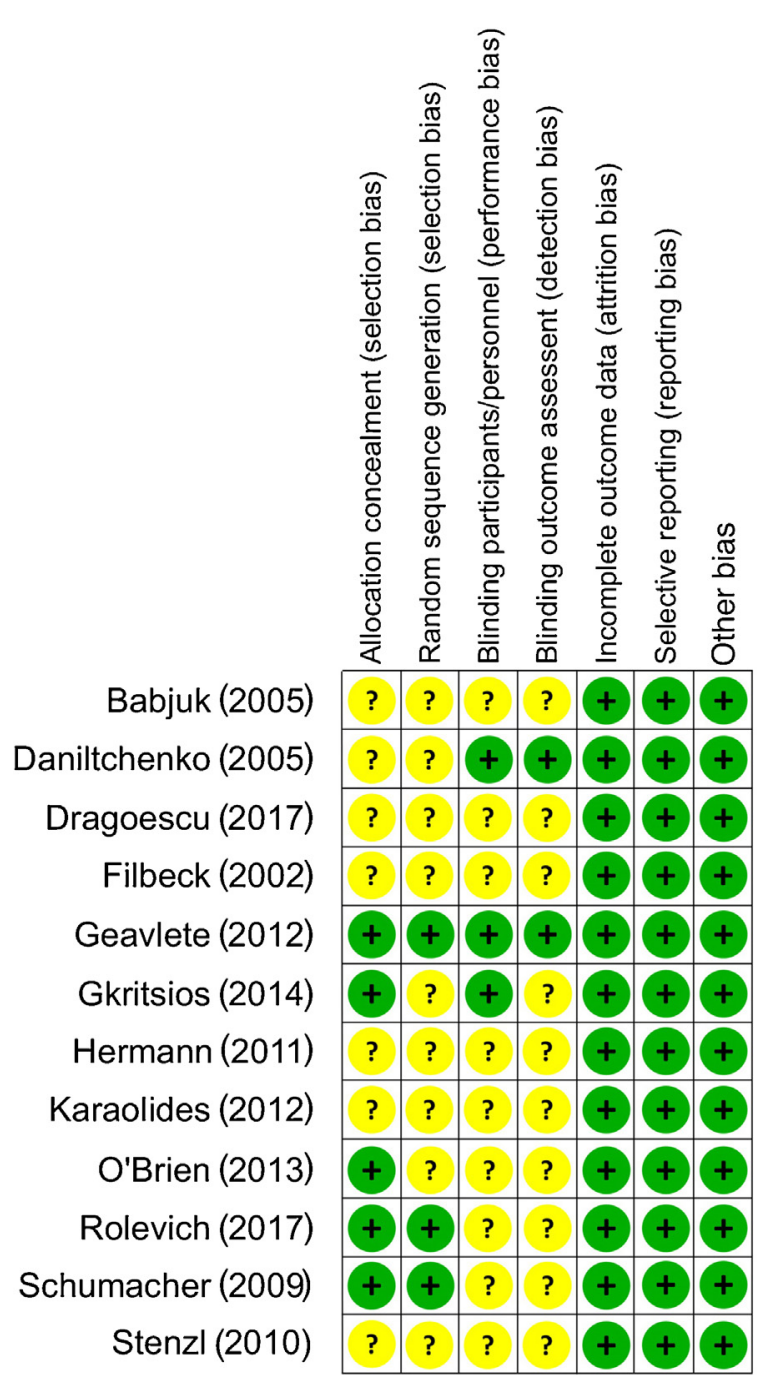

Fig. 4 - Risk of bias assessment.

single dose of IC. In the remaining studies $(n=6)$, a single dose of postoperative adjuvant IC was not administered, but patients were subsequently stratified on tumour characteristics and final pathology and risk-adapted intravesical therapy was offered [14,23,28-31]. This included some patients receiving intravesical bacillus Calmette-Guerin (BCG) or maintenance schedule of IC. It is also worth noting that, in the five studies where the intention was to give a single dose to all patients, not all were necessarily able to receive it, for example, in cases of suspected deep resection or on-going bleeding. In O'Brien et al's study [17], $37 \%$ in the PDD arm and $23 \%$ in the $\mathrm{WL}$ did not receive a dose. This figure, however, is not reported in any of the remaining four studies giving a universal single dose of chemotherapy.

The overall rates of recurrence at 12 mo in the PDD group in trials where single-dose IC was given were $9-33.8 \%$ (vs $18.9-43.7 \%$ for WL). The overall rates of recurrence at $24 \mathrm{mo}$ in the PDD group in trials where single-dose IC was given were $25-42.1 \%$ (vs $37.8-53.5 \%$ for $\mathrm{WL}$ ).
3.4.4. Tumour characteristics and recurrence patterns

Patients with high risk or multifocal disease have improved detection with PDD and hence intuitively would be the ones to benefit most. Six trials reported data for RFS based on tumour characteristics [14,17,24,25,28,30]. Filbeck et al [14] reported overall RFS for PDD versus WL of $89.6 \%$ versus $65.9 \%$ at 24 mo, with a more pronounced difference in PDD versus WL for high-risk tumours of $87.1 \%$ versus $40.4 \%$. Babjuk et al [28] also reported a more pronounced difference between PDD and WL for multifocal tumoursRFS at 24 mo of $39 \%$ versus $13 \%$ compared with the overall RFS of $40 \%$ versus $28 \%$ for PDD versus WL. This was also noted by Karaolides et al [25] who reported overall RFS at 18 mo of $82.5 \%$ for PDD versus $50.6 \%$ for WL, where for solitary tumours the RFS is equivocal (PDD $74.2 \%$ vs WL $76.7 \%$ ) compared with a distinct difference in the multifocal group (PDD 89.7\% vs WL 27.1\%). Geavlete et al [24] reported an overall $24-\mathrm{mo}$ RR of $31.2 \%$ for PDD versus $45.6 \%$ for $\mathrm{WL}$, again with an increased difference when comparing multifocal tumour cohorts only (PDD $35.4 \%$ vs $54 \%$ ). The remaining two studies did not report a similar recurrence pattern [17,30]. Schumacher et al [30] reported no evidence of a difference in overall RFS rates at 12 mo (PDD 55.9\% vs WL 55.1\%), and for high-risk disease only PDD was marginally worse (52.9\% vs WL 58.1\%); however, neither comparison was statistically significant $(p=0.689$ and $p=$ 0.346 , respectively). A similar picture was noted comparing uni/multifocal and low/high-grade tumours in the study of O’Brien et al [17], but was again not statistically significant.

\subsubsection{Quality assessment of studies}

The Cochrane risk of bias tool was utilised to assess quality. The majority of the studies were at a low/unclear risk of bias across assessed domains (Fig. 4). On GRADE evaluation, the certainty of the evidence was moderate for outcomes of RRs and RFS (Table 4). Certainty rating was downgraded by one level for risk of bias across both outcomes.

The funnel plots produced showed no evidence of publication bias but are limited by the small number of studies, and hence we accept that a risk publication bias may be present. The funnel plot for 12-mo risk ratio is shown in Figure 5. One method used to reduce the publication bias was the exclusion of multiple papers from the same patient cohort, analysing the most up-to-date publication.

\subsection{Discussion}

This review shows with a moderate GRADE certainty rating that PDD reduces RRs following initial TURBT compared with WL. The relative risks with PDD compared with WL were $0.73(0.60-0.88)$ at $12 \mathrm{mo}$ and $0.75(0.62-0.91)$ at $24 \mathrm{mo}$. At $60 \mathrm{mo}$, a $19.0-22.2 \%$ decrease in the rate of recurrence was observed. RFS following PDD was also increased (HR 1.14 [1.05-1.23] at $12 \mathrm{mo}$ and 1.25 [1.15-1.35] at $24 \mathrm{mo}$ ).

The diagnostic accuracy of PDD compared with WL is well established. A health technology assessment by Mowatt et al [35] included a systematic review and 
Table 4 - GRADE summary of certainty

\begin{tabular}{|c|c|c|c|c|c|c|}
\hline \multicolumn{3}{|c|}{ PDD TURBT vs WL TURBT } & & & & \\
\hline \multicolumn{3}{|c|}{$\begin{array}{l}\text { Patient or population: bladder cancer } \\
\text { Setting: hospital } \\
\text { Intervention: PDD TURBT } \\
\text { Comparison: WL TURBT }\end{array}$} & & & & \\
\hline \multirow{2}{*}{\multicolumn{2}{|c|}{ Outcomes }} & \multirow{2}{*}{$\begin{array}{c}\text { Number of } \\
\text { patients (studies) }\end{array}$} & \multirow{2}{*}{$\begin{array}{l}\text { Certainty of } \\
\text { evidence } \\
\text { (GRADE) }\end{array}$} & \multirow{2}{*}{$\begin{array}{l}\text { Relative effect } \\
\quad(95 \% \mathrm{CI})\end{array}$} & \multicolumn{2}{|c|}{ Anticipated absolute effects } \\
\hline & & & & & Risk with WL TURBT & Risk difference with recurrence rates \\
\hline \multirow[t]{3}{*}{$12 \mathrm{mo}$} & Combined & 833 (6 RCTs) & $\begin{array}{l}\oplus \oplus \oplus \hat{\mathrm{x}} \\
\text { Moderate }\end{array}$ & RR $0.73(0.45-0.82)$ & 387 per 1000 & 96 fewer per 1000 ( 146 fewer to 32 fewer) \\
\hline & HAL & 731 (5 RCTs) & $\begin{array}{l}\oplus \oplus \oplus \hat{\mathrm{x}} \\
\text { Moderate }\end{array}$ & RR $0.73(0.46-0.87)$ & 356 per 1000 & 105 fewer per 1000 ( 155 fewer to 46 fewer) \\
\hline & 5-ALA & $102(1 \mathrm{RCT})$ & $\begin{array}{l}\oplus \oplus \oplus \hat{\mathrm{x}} \\
\text { Moderate }\end{array}$ & RR $0.71(0.22-1.09)$ & 608 per 1000 & 176 fewer per 1000 ( 316 fewer to 46 more) \\
\hline \multirow[t]{3}{*}{$24 \mathrm{mo}$} & Combined & 539 (4 RCTs) & $\begin{array}{l}\oplus \oplus \oplus \hat{\mathrm{x}} \\
\text { Moderate }\end{array}$ & RR $0.75(0.62-0.91)$ & 504 per 1000 & 126 fewer per 1000 ( 191 fewer to 45 fewer) \\
\hline & HAL & 437 (3 RCTs) & $\begin{array}{l}\oplus \oplus \oplus \hat{\mathrm{X}} \\
\text { Moderate }\end{array}$ & RR $0.72(0.57-0.90)$ & 464 per 1000 & 130 fewer per 1000 ( 199 fewer to 46 fewer) \\
\hline & 5-ALA & $102(1 \mathrm{RCT})$ & $\begin{array}{l}\oplus \oplus \oplus \hat{\mathrm{x}} \\
\text { Moderate }\end{array}$ & RR $0.82(0.60-1.13)$ & 667 per 1000 & 120 fewer per 1000 ( 267 fewer to 87 more) \\
\hline $12 \mathrm{mo}$ & RFS & 1782 (9 RCTs) & $\begin{array}{l}\oplus \oplus \oplus \hat{\mathrm{x}} \\
\text { Moderate }\end{array}$ & HR $1.14(1.05-1.23)$ & & \\
\hline $24 \mathrm{mo}$ & RFS & 925 (5 RCTs) & $\begin{array}{l}\oplus \oplus \oplus \hat{\mathrm{X}} \\
\text { Moderate }\end{array}$ & HR 1.25 (1.15-1.35) & & \\
\hline \multicolumn{7}{|c|}{$\begin{array}{l}\text { 5-ALA = 5-aminolaevulinic acid; } \mathrm{CI}=\text { confidence interval; } \mathrm{GRADE}=\text { Grading of Recommendations Assessment, Development and Evaluation; HAL }= \\
\text { hexaminolaevulinate; } \mathrm{HR}=\text { hazard ratio; } \mathrm{PDD}=\text { photodynamic diagnosis; } \mathrm{RCT}=\text { randomised controlled trial; } \mathrm{RFS}=\text { recurrence-free survival; } \mathrm{RR}=\text { recurrence } \\
\text { rate; TURBT = transurethral resection of bladder tumour; } \mathrm{WL}=\text { white light. } \\
\text { Certainty rating downgraded by } 1 \text { for a high/unclear risk of bias. }\end{array}$} \\
\hline
\end{tabular}

meta-analysis and found overall PDD sensitivity of $92 \%$ versus $71 \%$ for WL. Whilst sensitivity was noted to be broadly similar for low-risk lesions (PDD 92\% vs WL 95\%), PDD was more sensitive at detecting high-risk lesions/CIS (PDD 89\% vs WL 52\%). RFS has previously been assessed in multiple earlier meta-analyses. Chou et al [18] looked at short term ( $<3 \mathrm{mo}$ ), intermediate (3-12 mo), and long-term ( $>12 \mathrm{mo}$ ) intervals. The longest follow-up in this study had a median duration of $42 \mathrm{mo}$. The authors reported reduced recurrence with PDD versus WL in the short term (ten trials, $n=1760$, RR 0.59, CI 0.4-0.88), intermediate term (six trials, $n=1150$, RR 0.7, CI 0.56-0.88), and long term (12 trials, $n=$ 2324, RR 0.81, CI 0.7-0.93). However, there was moderate heterogeneity $\left(\mathrm{I}^{2}=49 \%\right)$ in the long-term follow-up group, and the long-term group was further split into 12-mo follow-up (five trials) and $>12-$ mo (seven trials) groups, which showed RRs of 0.76 (CI 0.64-0.9) and 0.90 (0.69-1.17), respectively. Kausch et al [36] in 2010 performed a metaanalysis of three RCTs and concluded that RFS was $13.8-27 \%$ higher in the PDD group at 12 mo and 12-15\% higher at 24 mo. Mowatt et al [12] in 2011 also analysed RFS in two papers, both included by Kausch et al [36], and found that the 12- and 24-mo RFS was increased in the PDD group, though only statistically significantly at 24 mo (RR 1.37 [1.18-1.59]). Furthermore, Shen et al [37] in 2012 found that across 13 studies, there was no difference in RFS at 3 mo (RR $1.15, \mathrm{CI} 0.79-1.66, p=0.46$ ) or $12 \mathrm{mo}$ (RR 0.86, CI 0.7$1.06, p=0.16)$, though they noted that there was evidence of a reduction being present in the longer-term follow-up in some of the studies included. Our review provides an up-to- date analysis of the effects on RRs and RFS in the intermediate term, adhering to strict methodological standards. The inclusion of two newer studies, which were not included in prior reviews, with long-term follow-up periods allow for comment on the longer term.

From a practical perspective, as the use of PDD translates into a reduced $R R$ and improved RFS, we postulate that fewer resections for recurrent disease would be required, reducing overall cost in the long term. This is supported by previous economic assessments: a cost analysis by Burger et al [38] in 2007 reported that, despite the initial increased costs associated with PDD, there were savings overall in the 7-yr follow up period, with costs saved by PDD per patient per year of 168 euro. Whilst HAL is considerably costlier than 5-ALA, Dindyal et al [39] provided a UK-based cost analysis in 2008 for HAL PDD and with an estimated net saving of $£ 45500$ per 100 new NMIBC patients utilising PDD versus WL. Similarly, Malmström et al [40] in 2009 looked at a cost analysis for the use of HAL PDD in all new NMIBC patients in the Swedish health care system and estimated a saving of 1321716 Swedish Krona $(\sim £ 115000)$ in the 1 st year of implementation.

PDD appears to be safe and can be performed with minimal morbidity. In this systematic review, the overall rate of complications was low and two studies involving 376 patients reported no complications [14,17]. The majority of side effects experienced in the studies that did report adverse events were genitourinary in nature, with little or no additional side effects conferred by the use of PDD. This is consistent with previous literature: Cordeiro et al [41] 


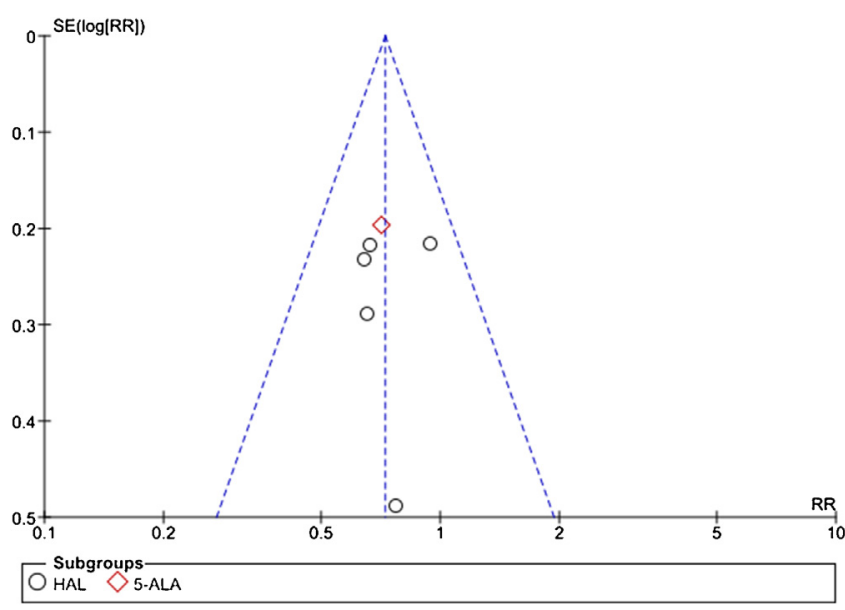

Fig. 5 - Funnel plot of 12-mo recurrence risk. 5-ALA = 5-aminolaevulinic acid; $H A L=$ hexaminolaevulinate; $R R=$ recurrence rate; $S E=$ standard error.

performed a literature review in 2013, which reported PDD to be well tolerated, regardless of the photosensitising agent, resulting in similar side effects to the standard WL procedure. A subsequent safety analysis looking solely at HAL performed by Witjes et al [34] in 2014 reported very little additional risk associated with the use of PDD.

The use of adjuvant IC may be a confounding factor in this analysis. It is well established that single-dose adjuvant IC reduces the rates of NMIBC recurrence, and therefore in studies where this was administered, we expected to see a lower RR and improved RFS. Sylvester et al [42] performed a meta-analysis in 2016, which showed that risk of recurrence was reduced by $35 \%$ with a single dose of adjuvant IC, except in patients with a high risk of recurrence. In all included studies after 2012, with the exception of Rolevich et al's [32] study, patients were scheduled to receive a single dose of adjuvant IC. The trial by Rolevich et al [32] had four arms, PDD \pm IC compared with WL \pm IC, with approximately $50 \%$ of each group given a single dose of doxorubicin. Comparing the arms with and without IC, there was no evidence of a difference in disease recurrence with an equivocal HR of 0.76 (CI 0.54-1.07, $p=0.11$ ). In contrast, a comparison of the PDD and WL arms showed a significantly decreased risk in disease recurrence in favour of PDD (HR 0.56; CI 0.39-0.80, p=0.001). However, it is unclear what effect the single dose will have on other studies where the effects on IC on RFS are not analysed. Likewise, the use of adjuvant intravesical BCG, which has been shown to reduce recurrence and progression of NMIBC, was variable throughout studies and inconsistently reported and is therefore another confounding factor.

There are limitations to our study. Clinical heterogeneity existed amongst the trials as regards the tumour presentation (primary or recurrent), tumour characteristics (size/ multifocality), final pathological grade, and the use of adjuvant treatment. These findings may affect RRs and RFS. Few data are available on the risk of progression, which is also another important consideration when managing NMIBC patients. We have attempted to minimise bias throughout the whole procedure, with rigorous search and selection criteria, standardised data extraction, and recalculation to evaluate the robustness of our findings. Given the marginal effect size in the efficacy trials included, the clinical utility in a real-world setting may be reduced, where it would be difficult to control for a number of confounders. In light of this, robust outcomes from trials focused on clinical effectiveness (real-world setting) that include longterm outcomes and account for intravesical treatments are awaited before stronger recommendations can be made. One such trial using this methodology is UK's NIHR-funded PHOTO trial (PMID: 31481549), which is due to report results in 2021.

\section{Conclusions}

In summary, this systematic review and meta-analysis provides moderate certainty of evidence that PDD reduces RRs and improves RFS for NMIBC compared with WL. This effect is observed over at least a 2-yr period and appears to persist for up to $5 \mathrm{yr}$. Results of pragmatic studies are awaited to inform recommendations.

Author contributions: Rakesh Heer had full access to all the data in the study and takes responsibility for the integrity of the data and the accuracy of the data analysis.

Study concept and design: Gravestock, Veeratterapillay, Heer. Acquisition of data: All authors.

Analysis and interpretation of data: All authors.

Drafting of the manuscript: All authors.

Critical revision of the manuscript for important intellectual content: All authors.

Statistical analysis: Gravestock, Veeratterapillay, Nambiar, Heer.

Obtaining funding: None.

Administrative, technical, or material support: Heer.

Supervision: Heer, Veeratterapillay.

Other: None.

Financial disclosures: Rakesh Heer certifies that all conflicts of interest, including specific financial interests and relationships and affiliations relevant to the subject matter or materials discussed in the manuscript (eg, employment/affiliation, grants or funding, consultancies, honoraria, stock ownership or options, expert testimony, royalties, or patents filed, received, or pending), are the following: None.

Funding/Support and role of the sponsor: None.

\section{Appendix A. Supplementary data}

Supplementary material related to this article can be found, in the online version, at doi:https://doi.org/10.1016/j. euros.2021.06.011.

\section{References}

[1] Richters A, Aben KKH, Kiemeney LALM. The global burden of urinary bladder cancer: an update. World J Urol 2020;38:1895-904. 
[2] Alfred Witjes J, Lebret T, Compérat EM, et al. Updated 2016 EAU guidelines on muscle-invasive and metastatic bladder cancer. Eur Urol 2017;71:462-75.

[3] Kamat AM, Hahn NM, Efstathiou JA, et al. Bladder cancer. Lancet (London England) 2016;388:2796-810.

[4] Sylvester RJ, van der Meijden AP, Oosterlinck W, et al. Predicting recurrence and progression in individual patients with stage Ta T1 bladder cancer using EORTC risk tables: a combined analysis of 2596 patients from seven EORTC trials. Eur Urol 2006;49, 466-5.

[5] D'Hallewin MA, Bezdetnaya L, Guillemin F. Fluorescence detection of bladder cancer: a review. Eur Urol 2002;42:417-25.

[6] Kennedy JC, Marcus SL, Pottier RH. Photodynamic therapy (PDT) and photodiagnosis (PD) using endogenous photosensitization induced by 5 -aminolevulinic acid (ALA): mechanisms and clinical results. J Clin Laser Med Surg 1996;14:289-304.

[7] Jocham D, Stepp H, Waidelich R. Photodynamic diagnosis in urology: state-of-the-art. Eur Urol 2008;53:1138-48.

[8] Schmidbauer J, Witjes F, Schmeller N, et al. Improved detection of urothelial carcinoma in situ with hexaminolevulinate fluorescence cystoscopy. J Urol 2004;171:135-8.

[9] Fradet Y, Grossman HB, Gomella L, et al. A comparison of hexaminolevulinate fluorescence cystoscopy and white light cystoscopy for the detection of carcinoma in situ in patients with bladder cancer: a phase III, multicenter study. J Urol 2007;178:68-73.

[10] Grossman HB, Gomella L, Fradet Y, et al. A phase III, multicenter comparison of hexaminolevulinate fluorescence cystoscopy and white light cystoscopy for the detection of superficial papillary lesions in patients with bladder cancer. J Urol 2007;178:62-7.

[11] Jichlinski P, Guillou L, Karlsen SJ, et al. Hexyl aminolevulinate fluorescence cystoscopy: new diagnostic tool for photodiagnosis of superficial bladder cancer-a multicenter study. J Urol 2003;170:226-9.

[12] Mowatt G, N'Dow J, Vale L, et al. Photodynamic diagnosis of bladder cancer compared with white light cystoscopy: systematic review and meta-analysis. Int J Technol Assess Health Care 2011;27:3-10.

[13] Rink M, Babjuk M, Catto JW, et al. Hexyl aminolevulinate-guided fluorescence cystoscopy in the diagnosis and follow-up of patients with non-muscle-invasive bladder cancer: a critical review of the current literature. Eur Urol 2013;64:624-38.

[14] Filbeck T, Pichlmeier U, Knuechel R, Wieland WF, Roessler W. Clinically relevant improvement of recurrence-free survival with 5-aminolevulinic acid induced fluorescence diagnosis in patients with superficial bladder tumors. J Urol 2002;168:67-71.

[15] Riedl CR, Daniltchenko D, Koenig F, Simak R, Loening SA, Pflueger H. Fluorescence endoscopy with 5-aminolevulinic acid reduces early recurrence rate in superficial bladder cancer. J Urol 2001;165:1121-3.

[16] Stenzl A, Burger M, Fradet Y, et al. Hexaminolevulinate guided fluorescence cystoscopy reduces recurrence in patients with nonmuscle invasive bladder cancer. J Urol 2010;184:1907-13.

[17] O’Brien T, Ray E, Chatterton K, Khan MS, Chandra A, Thomas K. Prospective randomized trial of hexylaminolevulinate photodynamic-assisted transurethral resection of bladder tumour (TURBT) plus single-shot intravesical mitomycin C vs conventional whitelight TURBT plus mitomycin C in newly presenting non-muscleinvasive bladder cancer. BJU Int 2013;112:1096-104.

[18] Chou R, Selph S, Buckley DI, et al. Comparative effectiveness of fluorescent versus white light cystoscopy for initial diagnosis or surveillance of bladder cancer on clinical outcomes: systematic review and meta-analysis. J Urol 2017;197(3 Pt 1):548-58.

[19] Higgins JPT, Thomas J, Chandler J, et al. Cochrane handbook for systematic reviews of interventions. ed. 2 Chichester, UK: John Wiley \& Sons; 2019.

[20] Moher D, Liberati A, Tetzlaff J, Altman DG, PRISMA Group. Preferred Reporting Items for Systematic Reviews and Meta-Analyses: the PRISMA statement. Int J Surg 2010;8:336-41.
[21] Sterne JAC, Savović J, Page MJ, et al. RoB 2: a revised tool for assessing risk of bias in randomised trials. BMJ 2019;366:14898.

[22] Guyatt GH, Oxman AD, Vist GE, et al. GRADE: an emerging consensus on rating quality of evidence and strength of recommendations. BMJ 2008;336:924-6.

[23] Hermann GG, Mogensen K, Carlsson S, Marcussen N, Duun S. Fluorescence-guided transurethral resection of bladder tumours reduces bladder tumour recurrence due to less residual tumour tissue in Ta/T1 patients: a randomized two-centre study. BJU Int 2011;108(8 Pt 2):E297-303.

[24] Geavlete B, Multescu R, Georgescu D, Jecu M, Stanescu F, Geavlete P. Treatment changes and long-term recurrence rates after hexaminolevulinate (HAL) fluorescence cystoscopy: does it really make a difference in patients with non-muscle-invasive bladder cancer (NMIBC). BJU Int 2012;109:549-56.

[25] Karaolides T, Skolarikos A, Bourdoumis A, et al. Hexaminolevulinate-induced fluorescence versus white light during transurethral resection of noninvasive bladder tumor: does it reduce recurrences. Urology 2012;80:354-9.

[26] Gkritsios P, Hatzimouratidis K, Kazantzidis S, Dimitriadis G, Ioannidis E, Katsikas V. Hexaminolevulinate-guided transurethral resection of non-muscle-invasive bladder cancer does not reduce the recurrence rates after a 2-year follow-up: a prospective randomized trial. Int Urol Nephrol 2014;46:927-33.

[27] Drăgoescu PO, Tudorache Ş, Drocaş AI, et al. Improved diagnosis and long-term recurrence rate reduction for non-muscle-invasive bladder cancer patients undergoing fluorescent hexylaminolevulinate photodynamic diagnosis. Rom J Morphol Embryol 2017;58:127983.

[28] Babjuk M, Soukup V, Petrík R, Jirsa M, Dvorácek J. 5-Aminolaevulinic acid-induced fluorescence cystoscopy during transurethral resection reduces the risk of recurrence in stage Ta/T1 bladder cancer. BJU Int 2005;96:798-802.

[29] Daniltchenko DI, Riedl CR, Sachs MD, et al. Long-term benefit of 5aminolevulinic acid fluorescence assisted transurethral resection of superficial bladder cancer: 5-year results of a prospective randomized study. J Urol 2005;174, 2129-33, discussion 2133.

[30] Schumacher MC, Holmäng S, Davidsson T, Friedrich B, Pedersen J, Wiklund NP. Transurethral resection of non-muscle-invasive bladder transitional cell cancers with or without 5-aminolevulinic acid under visible and fluorescent light: results of a prospective, randomised, multicentre study. Eur Urol 2010;57:293-9.

[31] Stenzl A, Penkoff H, Dajc-Sommerer E, et al. Detection and clinical outcome of urinary bladder cancer with 5 -aminolevulinic acidinduced fluorescence cystoscopy: a multicenter randomized, double-blind, placebo-controlled trial. Cancer 2011;117:938-47.

[32] Rolevich AI, Zhegalik AG, Mokhort AA, et al. Results of a prospective randomized study assessing the efficacy of fluorescent cystoscopyassisted transurethral resection and single instillation of doxorubicin in patients with non-muscle-invasive bladder cancer. World J Urol 2017;35:745-52.

[33] Soukup V, Čapoun O, Cohen D, et al. Risk stratification tools and prognostic models in non-muscle-invasive bladder cancer: a critical assessment from the European Association of Urology Non-muscleinvasive Bladder Cancer Guidelines Panel. Eur Urol Focus 2020;6:479-89.

[34] Witjes JA, Gomella LG, Stenzl A, Chang SS, Zaak D, Grossman HB. Safety of hexaminolevulinate for blue light cystoscopy in bladder cancer. A combined analysis of the trials used for registration and postmarketing data. Urology 2014;84:122-6.

[35] Mowatt G, Zhu S, Kilonzo M, et al. Systematic review of the clinical effectiveness and cost-effectiveness of photodynamic diagnosis and urine biomarkers (FISH, ImmunoCyt, NMP22) and cytology for the detection and follow-up of bladder cancer. 2010. In: NIHR Health 
Technology Assessment programme: Executive Summaries. Southampton, UK: NIHR Journals Library; 2003. https://www.ncbi.nlm. nih.gov/books/NBK56850/.

[36] Kausch I, Sommerauer M, Montorsi F, et al. Photodynamic diagnosis in non-muscle-invasive bladder cancer: a systematic review and cumulative analysis of prospective studies. Eur Urol 2010;57:595-606.

[37] Shen P, Yang J, Wei W, et al. Effects of fluorescent light-guided transurethral resection on non-muscle-invasive bladder cancer: a systematic review and meta-analysis. BJU Int 2012;110(6 Pt B):E209-15.

[38] Burger M, Zaak D, Stief CG, et al. Photodynamic diagnostics and noninvasive bladder cancer: is it cost-effective in long-term application? A Germany-based cost analysis. Eur Urol 2007;52:142-7.

[39] Dindyal S, Nitkunan T, Bunce CJ. The economic benefit of photodynamic diagnosis in non-muscle invasive bladder cancer. Photodiagnosis Photodyn Ther 2008;5:153-8.
[40] Malmström PU, Hedelin H, Thomas YK, Thompson GJ, Durrant H, Furniss J. Fluorescence-guided transurethral resection of bladder cancer using hexaminolevulinate: analysis of health economic impact in Sweden. Scand J Urol Nephrol 2009;43:192-8.

[41] Cordeiro ER, Anastasiadis A, Bus MT, Alivizatos G, de la Rosette JJ, de Reijke TM. Is photodynamic diagnosis ready for introduction in urological clinical practice. Expert Rev Anticancer Ther 2013;13:669-80.

[42] Sylvester Rj, Oosterlinck W, Holmang S, et al. Systematic review and individual patient data meta-analysis of randomized trials comparing a single immediate instillation of chemotherapy after transurethral resection with transurethral resection alone in patients with stage pTa-pT1 urothelial carcinoma of the bladder: which patients benefit from the instillation. Eur Urol 2016;69:231-44. 